\title{
Alternative Sample Preparation Method for Large-Area Cross-Section View Observation of Lithium Ion Battery
}

\author{
Ji-Young Kim ${ }^{1}$, Young Woo Jeong ${ }^{1}$, Hye Young $\mathrm{Cho}^{1}$, Hye Jung Chang ${ }^{1,2, *}$ \\ ${ }^{1}$ Advanced Analysis Center, Korea Institute of Science and Technology (KIST), Seoul 02792, Korea \\ ${ }^{2}$ Department of Nanomaterials Science and Technology, University of Science and Technology, Daejeon 34113, Korea
}

*Correspondence to:

Chang $\mathrm{HJ}$,

Tel: +82-2-958-5365

Fax: +82-2-958-6974

E-mail: almacore@kist.re.kr

Received June 12, 2017

Revised June 28, 2017

Accepted June 30, 2017

\begin{abstract}
Drastic development of ubiquitous devices requires more advanced batteries with high specific capacitance and high rate capability. Large-area microstructure characterization across the stacks of cathode, electrolyte and anode might reveal the origin of the instability or degradation of batteries upon cycling charge. In this study, sample preparation methods to observe the cross-section view of the electrodes for battery in SEM and several imaging tips are reviewed. For an accurate evaluation of the microstructure, ion milling which flats the surface uniformly is recommended. Pros and cons of cross-section polishing (CP) with Ar ion and focused ion beam (FIB) with Ga ion were compared. Additionally, a modified but new cross-section milling technique utilizing precision ion polishing system (PIPS) which can be an alternative method of CP is developed. This simple approach will make the researchers have more chances to prepare decent large-area cross-section electrode for batteries.
\end{abstract}

Key Words: Li-ion batteries, Microstructure characterization, Scanning electron microscope (SEM), Ar milling, Cross-section plane

\section{INTRODUCTION}

A secondary battery refers to a device that can repeatedly store electric energy into a form of chemical energy and use it (Palacin, 2009). Among a variety of secondary batteries, lithium-ion batteries have a wide range of applications, including being used in cellphones, laptops, and other portable electronic devices as well as hybrid cars, energy storage systems, and other medium-to-large systems (Goodenough \& Park, 2013). The development of lithiumion secondary batteries mainly focuses on the improvement of anode and cathode materials and exploring new materials, which determine the battery's performance (Chen, 2013; Koksbang et al., 1996; Wang et al., 2015; Zhang et al., 2015; Zou et al., 2015). Further, understanding the reaction and degradation mechanism of the anode and cathode materials is critical in material development, toward which various analysis technologies need to be introduced (Liu et al.,
2014; Vanimisetti \& Ramakrishnan, 2012). Among the key technologies in the secondary battery material analysis, it is expected that there will be many applications of the microstructural analysis technology of materials using an electronic microscope, together with the research on active materials using an X-ray synchrotron. In recent years, in situ techniques are used to analyze the electronic and structural changes during the charge-discharge or heating process (Chen et al., 2011; Chung et al., 2007; Nam et al., 2013; Yoon et al., 2007, 2014).

Scanning electron microscope (SEM) can be used for identifying cracks caused by the degradation characteristics of the materials during the charge-discharge process or analyzing the structural change due to the volume expansion of electrodes (Ebner et al., 2013; Liu et al., 2014). SEM is the easiest analysis method to observe the failed section; however, it is difficult to analyze and quantify the size of particles or pores because the observed section is not even (Zhang

(a) This is an open-access article distributed under the terms of the Creative Commons Attribution Non-Commercial License (http://creativecommons.org/licenses/by-nc/4.0) which permits unrestricted noncommercial use, distribution, and reproduction in any medium, provided the original work is properly cited.

Copyrights @ 2017 by Korean Society of Microscopy 
et al., 2000). Moreover, the sample preparation is required to smooth out the uneven section during the chemical composition analysis using the energy-dispersive spectrum or an electron probe micro-analyzer. There are, however, a number of limitations in preparing for bulk specimens of lithium-ion secondary batteries for image analysis. As most anode and cathode materials are susceptible to moisture and have a porous structure, mechanical polishing cannot be used as the large-area cross-section sampling preparation method. Instead, ion-milling method using a cross-section polisher (CP) and a focused ion beam (FIB) can be used (Ebner et al., 2013; Indrikova et al., 2015; Liu et al., 2016; Otoyama et al., 2016).

FIB is often useful in observing specific areas by finely controlling the $\mathrm{Ga}^{+}$ion source. However, it has limitations in that the device operation is complex and expensive. On the contrary, $\mathrm{CP}$, which uses $\mathrm{Ar}^{+}$gas as its ion source, sets the milling location using an optical microscope. While it cannot perform milling on a specific area, its large beam size allows for large-area milling. Therefore, it is extremely important to select a cross-section sampling method that meets the aims of the analysis of secondary batteries used (Deng et al., 2017; Indrikova et al., 2015; Kawaguchi et al., 2017; Kim et al., 2008; Otoyama et al., 2016). This study explained a sampling method for cross-section imaging analysis based on the research objectives by conducting a comparative analysis of the sample analysis results using CP and FIB, which are two most widely used cross-section sampling methods for SEM imaging analysis. Furthermore, it introduced the large-area cross-section milling technique using Precision Ion Polisher System (PIPS), which is commonly used in the final milling stage of the TEM specimen production, as an alternative to the CP.

\section{MATERIALS AND METHODS}

\section{Fabrication of Electrode}

The typical cathode materials were used to compare cross-section images. The $\mathrm{Li}\left(\mathrm{Ni}_{1 / 3} \mathrm{Co}_{1 / 3} \mathrm{Mn}_{1 / 3}\right) \mathrm{O}_{2}$ or $\mathrm{LiFePO}$ electrodes were prepared by mixing of active materials, conducting agent, and binder. Detailed procedures for the fabrication of electrodes were as described previously (Chang et al., 2006; Kang et al., 2014). The ultimately obtained slurry was casted onto Al foil. The commercial Li-ion coin cell (PD 2032, Korea Powercell) were also used. The anode in coincell was used to compare ion-milled region and fractured region. The cathode in coincell was used to compare the secondary electron image and backscattered electron image.

\section{Cross-Section Milling}

First, the study performed cross-section milling of electrodes using a CP (E-3500; Hitachi Inc., Japan), which is widely used for large-area milling. The cross-section of an electrode was cut to $1 \times 1 \mathrm{~cm}^{2}$ or less and was fixed with carbon tape so that approximately $1 \sim 2 \mathrm{~mm}$ of the sample extended out from the stub. The tungsten mask was attached to the specimen stage unit, and the sample stub was fixed such that the surface to which the specimen was attached faced the mask. Using the optical microscope, the milling position of the specimen was adjusted to the incident beam height. Milling was performed for $8 \mathrm{~h}$ under the following conditions: acceleration voltage of $6 \mathrm{keV}$; discharge voltage of $4 \mathrm{keV}$; and $\mathrm{Ar}$ gas flow of $1.5 \mathrm{~cm}^{2} /$ min. For comparison, ion milling was performed using dualbeam FIB (Helios NanoLab600; FEI Co., USA). By tilting the specimen in the direction perpendicular to the ion beam, a Pt protection layer was deposited onto the surface of region of interest. The incident ion beam had an accelerating voltage of $30 \mathrm{kV}$ and a current of $2.8 \mathrm{nA}$. As an alternative method to CP, the study used PIPS (691; Gatan Inc., USA), and a homemade mount was used for mounting bulk specimens. A detailed explanation will be given in Large Area Cross-Section Milling by PIPS in Results and Discussion.

\section{Microstructure Characterization}

After cross-sectional milling, cross-sectional high-resolution images of large area of the specimen were acquired using a scanning electron microscope(TENEO VS ${ }^{\mathrm{TM}}$ SEM; FEI Co.) with MAPS 2.1 software (FEI Co.), an automated image acquisition and stitching software. The MAPS allows the automatic acquisition and reconstruction of several highresolution images based on the predetermined configurations. The images were acquired at a magnification of over 10,000 times, with an acceleration voltage of $2 \mathrm{keV}$, beam current of $12.5 \mathrm{pA}$, and dwell time at $3 \mu \mathrm{s}$. The number of tiles was set to

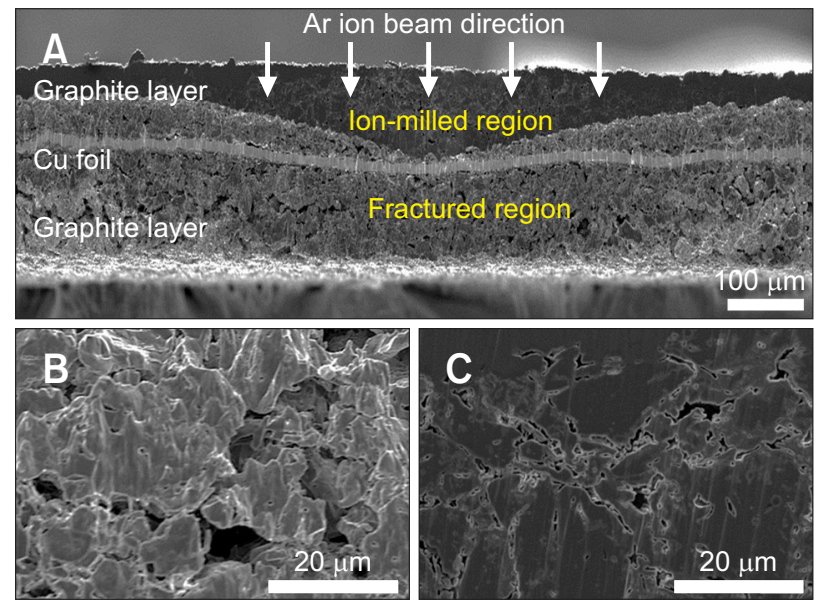

Fig. 1. (A) Cross-sectional SEM images of anode in commercial coin cell consisting of graphite and $\mathrm{Cu}$ foil. Upper graphite layer is ion milled, but $\mathrm{Cu}$ foil and lower graphite layer is not milled yet. Magnified images obtained from (B) fractured region and (C) ion-milled region compare the surface roughness. 
$\mathrm{X} \times \mathrm{Y}=10 \times 4$; for accurate stitching, the overlap area of $\mathrm{X}$ and $\mathrm{Y}$ was set to $10 \%$. All the acquired images were auto-aligned later to produce one high-resolution image.

\section{RESULTS AND DISCUSSION}

\section{Necessities of Ion Milling Sample Preparation Method}

The importance of performing ion milling without using a knife to fracture the sample when observing lithium-ion secondary battery materials is shown in Fig. 1. Fig. 1A shows the large-area microstructure of an anode material with graphite layers at both ends of the $\mathrm{Cu}$ foil; the upper part was ion-milled with $\mathrm{CP}$ while the lower part was remained as cutting plane with a knife. The magnified images of the
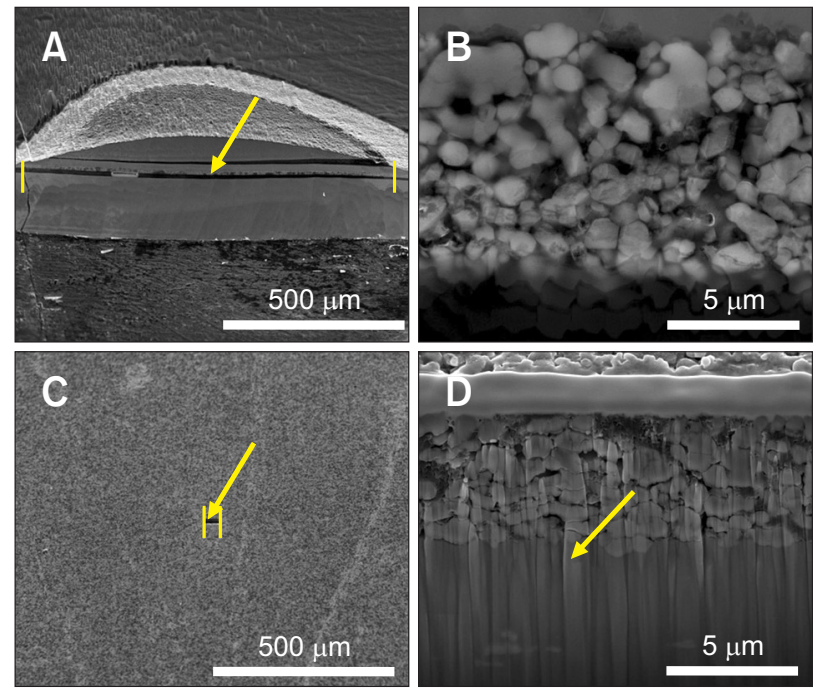

Fig. 2. Cross-section $\mathrm{Li}\left(\mathrm{Ni}_{1 / 3} \mathrm{Co}_{1 / 3} \mathrm{Mn}_{1 / 3}\right) \mathrm{O}_{2}$ samples are prepared by $\mathrm{CP}$ in $(\mathrm{A}, \mathrm{B})$ and FIB in (C, D). Low magnification SEM images in (A) and (C) show the available regions in CP and FIB. Magnified images in (B) and (D) show that milling artefact in porous materials, so called curtain effect, is less in sample prepared with CP. fractured area and ion-milled area are compared in Fig. 1B and $\mathrm{C}$, respectively. While the sample preparation is very simple and easy for the fracture method using a knife, it can damage the interior of the specimen; furthermore, due to the rough surface and micro-crack caused by cutting the section, the pore size is distorted. On the contrary, the cross-section image of the electrode with CP is relatively smoother and does not show any cracks, allowing for detailed analysis of the pore size. As a result, the pore size in (Fig. 1B) appears to be bigger than that in (Fig. 1C). Therefore, sample preparation using ion milling is required for accurate analysis of the microstructure of the material.

\section{Pros and Cons of FIB Milling}

Besides CP, FIB is also used for the SEM cross-section analysis of secondary battery materials. The biggest difference between the two sample preparation techniques is the size of the area that can be analyzed. Fig. 2A and C show the secondary electron images of the specimens with cross-section milling using $\mathrm{CP}$ and FIB, respectively. When milling is done for the same duration, the cross-section analysis using FIB allows only for areas in several tens of $\mu \mathrm{m}$ scale whereas CP can allow for the analysis of areas as large as several $\mathrm{mm}$. Therefore, if the target area is large, CP is more proper than FIB. FIB has limitations not only in terms of the width of the crosssectional sampling area but also in terms of the milling depth. In the case of electrodes whose thickness is within several micrometers, the sampling using FIB offers only a restricted range of depth that displays uniform milling, and only the near-surface part of electrodes can be analyzed. Consequently, the results cannot represent the entire electrode material. Second, the two techniques are different in the degree of curtain effect, which appears with the milling of porous materials. Fig. $2 \mathrm{~B}$ and $2 \mathrm{D}$ compare high-magnification SE images prepared with $\mathrm{CP}$ and FIB, respectively. Fig. 2D does not clearly show the shape and size of the particles due to the curtain effect. To minimize the curtain effect, the acceleration
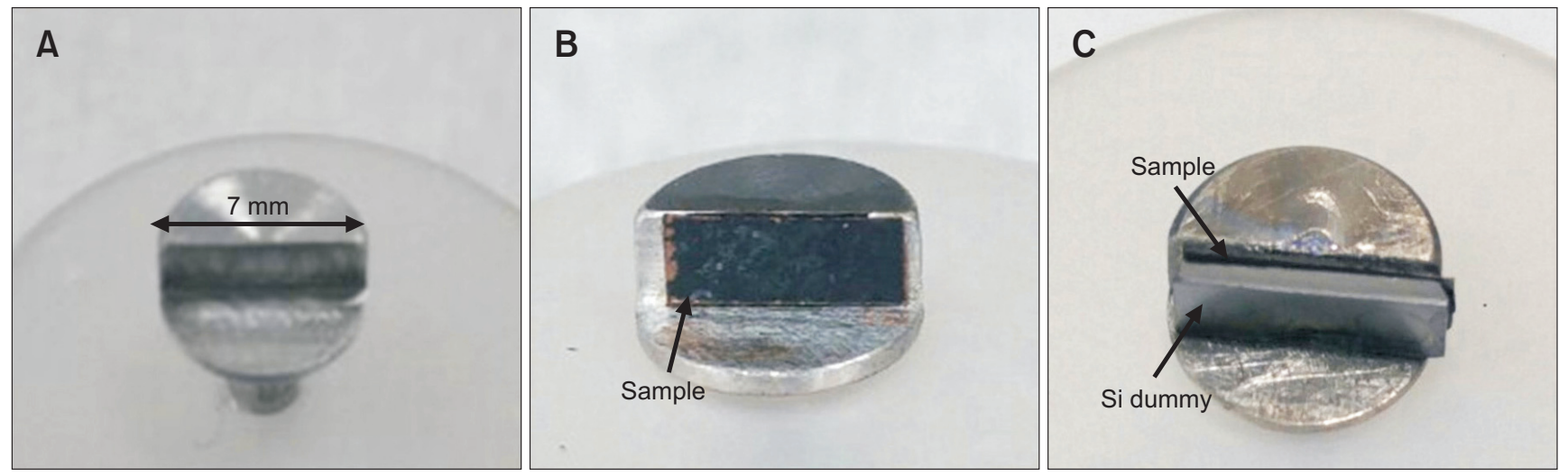

Fig. 3. (A) Modified PIPS mount for cross-section milling of bulk sample; (B) and (C) procedure of sample loading with Si mask on the mount. 
voltage or the change in the milling angle can be controlled; however, due to the characteristics of FIB milling, it is difficult to remove curtain effect completely. On the contrary, the SE image with CP does not show much curtain effect, and thus the boundary, size, and shape of particles can be clearly observed. Therefore, considering the limitations of large-area analysis, curtain effect, and high cost, FIB is considered more suitable for selective local area analysis rather than general cross-section analysis of secondary batteries.

\section{Large-Area Cross-Section Milling by PIPS}

As discussed above, CP is considered more useful in large-area cross-section analysis. However, not many research groups are equipped with CP devices. However, Ar milling system of PIPS, which is used for preparing TEM samples, are relatively common; therefore, this study developed a large-area milling method using PIPS (Houben et al., 2016a, 2016b). It is one of the systems that ultimately create thin, wedge-type TEM samples by milling polished samples with Ar ion beam. Generally, thin specimens that were mechanically polished below $50 \mu \mathrm{m}$ are mounted on the duopost, and the ion beam is applied to the specimen from below and above it for milling. However, battery materials for cross-section analysis are bulk samples whose size ranges up to several millimeters, thereby requiring a special mount to load the specimens on PIPS. A mount shown in Fig. 3A was attached to that of the mount, adjusting the sample height to the top of the mount. To prevent the ion beam from making direct contact with the specimen, a Si wafer mask was attached to the front of the specimen, as shown in Fig. 3C. After the mount was loaded on the stage, two ion guns were set to the direction of the top of the specimen, and the stage was set to the single-beam mode. The acceleration voltage for the Ar ion beam was set to 4.0 $\mathrm{keV}$, and its angle was maintained at $3^{\circ}$.

Fig. 4 shows the comparison of the angle between the specimen and the incident ion beam for CP and PIPS. In the case of CP, the gun-mask-sample is vertically aligned, and the milling range can be controlled by swinging or shifting the stage. On the contrary, in the case of PIPS, two Ar guns are horizontally set at an angle of $120^{\circ}$ and the section of the specimen is mounted in-plane. The angle of the gun applied to the specimen can be controlled between $0^{\circ} \pm 10^{\circ}$. In the single-beam mode among the three stage modes, the stage rotates as shown in the inset of Fig. $4 \mathrm{~B}$, and the ion beam is turned on only at a specific area (milling only one sector of the sample) where the gun-mask-sample is aligned. Fig. 5 shows the resulting SEM image of the cross-section milled electrode material. It can be determined that a relatively large are of cross-section milling up to approximately 1.61 $\mathrm{mm}$ was performed. Fig. $5 \mathrm{~B}$ and $\mathrm{C}$ are the medium- and high-magnification SEM images in the region of interest, and although the milled surface is smooth and is a porous material, no curtain effect was observed. PIPS is advantageous in reducing the curtain effect because the specimen is rotated and there is a small angle between the specimen and the ion beam. As a result, the pore inside the active material or the size of the active material particles could be accurately observed. Furthermore, the thickness of the electrode section could be accurately analyzed. Therefore, the use of PIPS as an alternative to $\mathrm{CP}$ or FIB, which have been used widely for the cross-section sampling of the electrode material of lithiumion secondary batteries, is expected to offer a sound sample
A

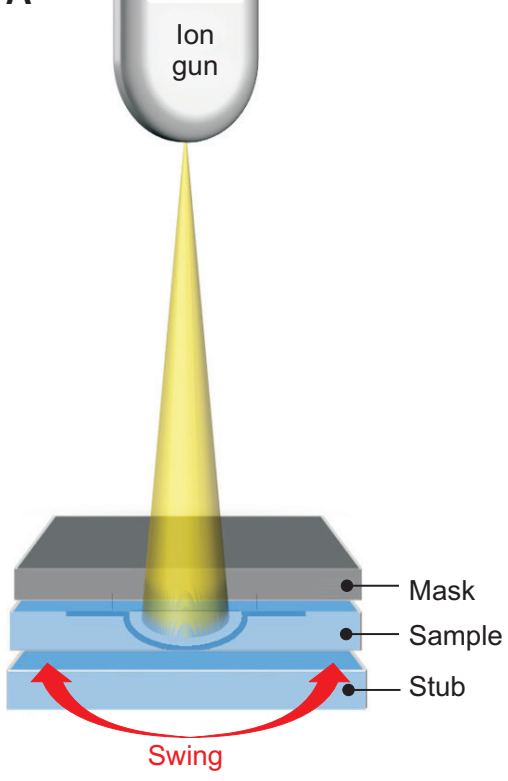

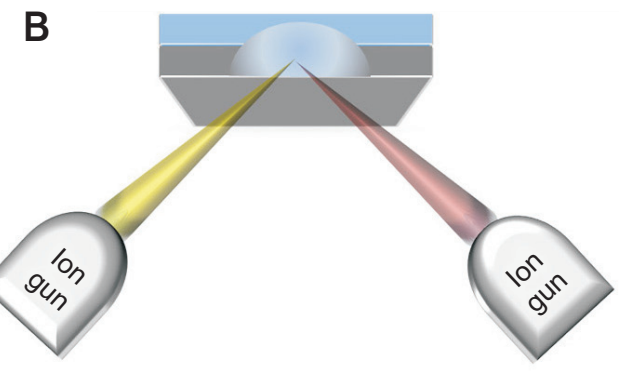

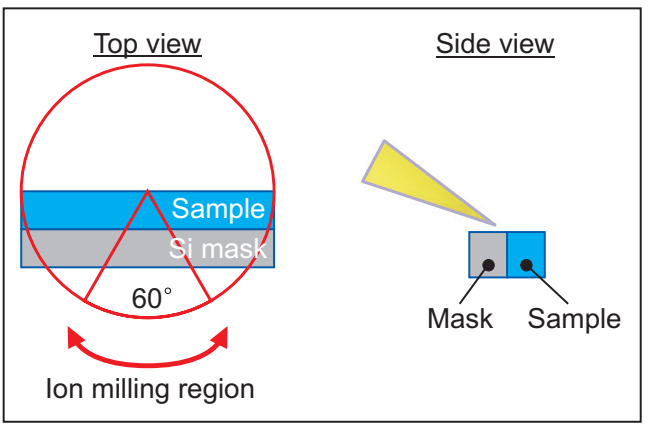

Fig. 4. Schematics of (A) CP and (B) PIPS for cross-section milling of bulk sample showing the alignment of ion gun-masksample-stub. The inset box in (B) shows milling direction and area in single beam mode of PIPS. 

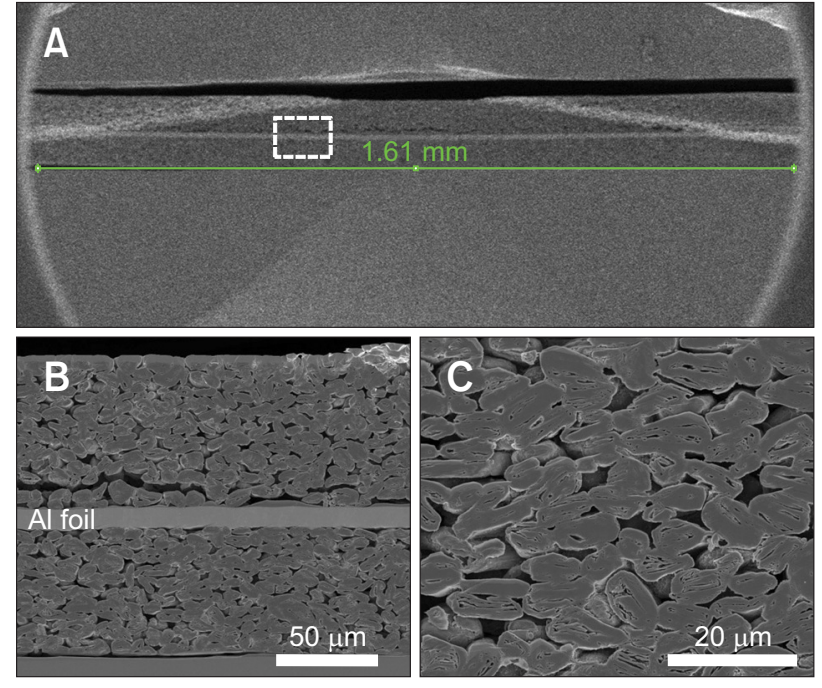

Fig. 5. (A) Low magnification SEM image of $\mathrm{LiFePO}_{4}$ shows milling area by PIPS. The milled region is large upto $1.6 \mathrm{~mm}$ in diameter. (B) and (C) are magnified image in the box area in (A), which shows flat and smooth surface, visualizing the interface and particle size successfully.

preparation method without surface roughness artifacts in a sufficiently large area with relative low costs and less effort in the optimization of equipment operation conditions.

\section{SEM Imaging Tips for Battery Materials}

While the use of CP would result in a relatively weaker curtain effect, it is difficult to completely prevent the effect caused by pores when cross-section milling materials with a porous structure (Fig. 2). As backscattered electron (BSE) images are less sensitive to surface roughness, it can avoid the distortion of the microstructure analysis due to the curtain effect resulting from $\mathrm{CP}$ process. Additionally, to determine the microstructure of electrodes with heterogeneous materials (metal oxide and carbon), it is recommended to use the BSE mode that can maximize contrast. Electrodes are composed of three materials: active materials, conductive materials, and binder. The distribution of three components-active materials such as $\mathrm{LiCoO}_{2}, \mathrm{LiFePO}_{4}$, conducting agents like carbon, and binder-are key factors in the electrical and chemical properties such as long cycle life and high-efficiency properties of secondary batteries (Dominko et al., 2003; Feng et al., 2007; Guy et al., 2004; Xiao et al., 2012). As the contrast in the BSE image analysis is determined by the atomic number of the materials that comprise the sample, the BSE image analysis is suitable for clearly distinguishing the distribution of conducting agent comprising light elements and active materials that include relatively heavier elements within the electrode (Park et al., 2004; Zhang et al., 2006). Fig. $6 \mathrm{~B}$ shows the BSE image acquired from the identical specimen in Fig. 6A. It demonstrates that particles of active material
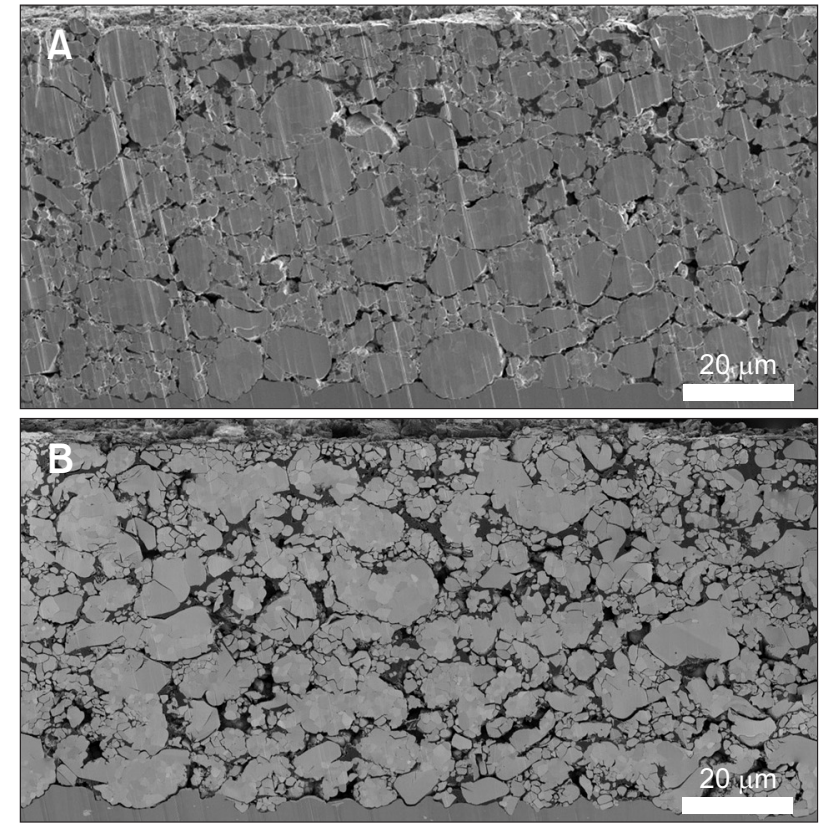

Fig. 6. Cross-sectional images of cathode in commercial coin cell in (A) secondary electron mode and (B) back scattered electron mode.

having a bright contrast are uniformly distributed without agglomeration. Furthermore, the image of the interface between the particles of active material and conducting agent is clearly acquired, through which the shape and size of the particles can be accurately determined. Therefore, even when a researcher uses the identical cross-section SEM analyses, it is important to select an appropriate SEM image mode depending on the goal of the particular research.

Fig. 6 shows the results of the reconstruction of the 40 highresolution images in the milling area after the cross-section sampling of the area over $1 \mathrm{~mm}$, using MAPS 2.1 software. MAPS allows for creating large-area high-resolution images by the automated acquisition and reconstruction of tens of high-resolution SEM images by tiling and stitching. Such analysis results demonstrate that the proposed method enables analysis of large-area images of the electrodes and produces highly representative and reliable analysis results. Furthermore, as the technique allows for microstructural analysis of specific areas, it is highly useful to determine what types of change occur in which area of the sample when comparing the changes in the electrode structure before and after the charge-discharge process.

\section{CONCLUSIONS}

In this study, sample preparation methods to observe the cross-section view of the electrode of battery in SEM are reviewed. It is important to make flat and smooth surface to 
evaluate the intrinsic microstructure including size and shape of the particles, microcracks, solid electrolyte interphase (SEI) layer, and distribution of the particles/binder/pores. Specific region can be observed after cross-section milling using FIB. CP makes the sample flat in large area and the curtain effect which is difficult to avoid in FIB can be minimized by swinging the sample stage. Here, we developed alternative ion milling methods using PIPS, which provides similar output with CP. Millimeter scale milling was available and no curtain effect was observed due to sample rotation and a small angle between the sample and the incident beam even in a porous materials. It is expected that this simple approach to modify the PIPS mount will let many researchers who are available to access to the PIPS also prepare decent large-area cross-section view samples.

Additionally, two SEM imaging tips for electrodes are mentioned. BSE imaging is useful since that gives chemical composition sensitive contrast distinguishing the oxide particle and binder even in samples with rough surface, which can be induced by fracture method or ion milling in porous battery electrode materials. Tiling and stitching method let us analyze the microstructure generally and specifically at one time, which could be useful evaluate the microstructural evolution upon charge cycling.

\section{CONFLICT OF INTEREST}

No potential conflict of interest relevant to this article was reported.

\section{ACKNOWLEDGMENTS}

This work was supported by a grant of Korea Institute of Science and Technology (KIST) through 2V05210 and 2E27090.

\section{REFERENCES}

Chang H H, Chang C C, Wu H C, Guo Z Z, Yang M H, Chiang Y P, Sheu S, and Wu N L (2006) Kinetic study on low-temperature synthesis of LiFePO4 via solid-state reaction. J. Power Sources 158, 550-556.

Chen D, Indris S, Schulz M, Gamer B, and Monig R (2011) In situ scanning electron microscopy on lithium-ion battery electrodes using an ionic liquid. J. Power Sources 196, 6382-6387.

Chen J J (2013) Recent progress in advanced materials for lithium ion batteries. Materials 6, 156-183.

Chung K Y, Yoon W S, McBreen J, Yang X Q, Oh S H, Shin H C, Cho W I, and Cho B W (2007) In situ X-ray diffraction studies on the mechanism of capacity retention improvement by coating at the surface of $\mathrm{LiCoO}_{2}$. J. Power Sources 174, 619-623.

Deng $Y$ Y, He Z Y, Cao Q, Jing B, Wang X Y, and Peng X X (2017) A novel high-performance electrospun thermoplastic polyurethane/ poly(vinylidene fluoride)/polystyrene gel polymer electrolyte for lithium batteries. Acta Chim. Slov. 64, 95-101.

Dominko R, Gaberscek M, Drofenik J, Bele M, Pejovnik S, and Jamnik J (2003) The role of carbon black distribution in cathodes for $\mathrm{Li}$ ion batteries. J. Power Sources. 119, 770-773.

Ebner M, Marone F, Stampanoni M, and Wood V (2013) Visualization and quantification of electrochemical and mechanical degradation in li ion batteries. Science 342, 716-720.

Feng C Q, Chew S Y, Guo Z P, Wang J Z, and Liu H K (2007) An investigation of polypyrrole-LiV308 composite cathode materials for lithium-ion batteries. J. Power Sources 174, 1095-1099.

Goodenough J B and Park K S (2013) The Li-ion rechargeable battery: a perspective. J. Am. Chem. Soc. 135, 1167-1176.

Guy D, Lestriez B, and Guyomard D (2004) New composite electrode architecture and improved battery performance from the smart use of polymers and their properties. Adv. Mater. 16, 553-557.

Houben M E, Barnhoorn A, Lie-A-Fat J, Ravestein T, Peach C J, and Drury M R (2016) Microstructural characteristics of the Whitby Mudstone Formation (UK). Mar. Petrol. Geol. 70, 185-200.
Houben M E, Barnhoorn A, Wasch L, Trabucho-Alexandre J, Peach C J, and Drury M R (2016) Microstructures of Early Jurassic (Toarcian) shales of Northern Europe. Int. J. Coal. Geol. 165, 76-89.

Indrikova M, Grunwald S, Golks F, Netz A, Westphal B, and Kwade A (2015) The morphology of battery electrodes with the focus of the conductive additives paths. J. Electrochem. Soc. 162, A2021-A2025.

Kang K S, Choi S, Song J, Woo S G, Jo Y N, Choi J, Yim T, Yu J S, and Kim Y J (2014) Effect of additives on electrochemical performance of lithium nickel cobalt manganese oxide at high temperature. J. Power Sources 253, 48-54.

Kawaguchi T, Nakamura H, and Watano S (2017) Parametric study of dry coating process of electrode particle with model material of sulfide solid electrolytes for all-solid-state battery. Powder Technol. 305, 241249.

Kim H, Han B, Choo J, and Cho J (2008) Three-dimensional porous silicon particles for use in high-performance lithium secondary batteries. Angew. Chem. Int. Edit. 47, 10151-10154.

Koksbang R, Barker J, Shi H, and Saidi M Y (1996) Cathode materials for lithium rocking chair batteries. Solid State Ionics. 84, 1-21.

Liu H S, Foster J M, Gully A, Krachkovskiy S, Jiang M, Wu Y, Yang X Y, Protas B, Goward G R, and Botton G A (2016) Three-dimensional investigation of cycling-induced microstructural changes in lithiumion battery cathodes using focused ion beam/scanning electron microscopy. J. Power Sources 306, 300-308.

Liu N, Lu Z D, Zhao J, McDowell M T, Lee H W, Zhao W T, and Cui Y (2014) A pomegranate-inspired nanoscale design for large-volume-change lithium battery anodes. Nat. Nanotechnol. 9, 187-192.

Nam K W, Bak S M, Hu E Y, Yu X Q, Zhou Y N, Wang X J, Wu L J, Zhu Y M, Chung K Y, and Yang X Q (2013) Combining in situ synchrotron X-ray diffraction and absorption techniques with transmission electron microscopy to study the origin of thermal instability in overcharged cathode materials for lithium-ion batteries. Adv. Funct. Mater. 23, 1047-1063. 
Otoyama M, Ito Y, Hayashi A, and Tatsumisago M (2016) Raman imaging for $\mathrm{LiCoO}_{2}$ composite positive electrodes in all-solid-state lithium batteries using $\mathrm{Li}_{2} \mathrm{~S}-\mathrm{P}_{2} \mathrm{~S}_{5}$ solid electrolytes. J. Power Sources 302 , 419-425.

Palacin M R (2009) Recent advances in rechargeable battery materials: a chemist's perspective. Chem. Soc. Rev. 38, 2565-2575.

Park K S, Son J T, Chung H T, Kim S J, Lee C H, Kang K T, and Kim H G (2004) Surface modification by silver coating for improving electrochemical properties of $\mathrm{LiFePO}_{4}$. Solid State Commun. 129, 311-314.

Vanimisetti S K, Ramakrishnan N (2012) Effect of the electrode particle shape in Li-ion battery on the mechanical degradation during chargedischarge cycling. P. I. Mech. Eng. C-J Mec. 226, 2192-2213.

Wang F X, Xiao S Y, Li M X, Wang X W, Zhu Y S, Wu Y P, Shirakawa A, and Peng J (2015) A nanocomposite of $\mathrm{Li}_{2} \mathrm{MnO}_{3}$ coated by $\mathrm{FePO}_{4}$ as cathode material for lithium ion batteries. J. Power Sources 287, 416-421.

Xiao L F, Cao Y L, Xiao J, Wang W, Kovarik L, Nie Z M, and Liu J (2012) High capacity, reversible alloying reactions in SnSb/C nanocomposites for Na-ion battery applications. Chem. Commun. $\mathbf{4 8 ,}$ 3321-3323.
Yoon W S, Chung K Y, McBreen J, Fischer D A, and Yang X Q (2007) Electronic structural changes of the electrochemically Li-ion deintercalated $\mathrm{LiNi}_{0.8} \mathrm{CO}_{0.15} \mathrm{Al}_{0.05} \mathrm{O}_{2}$ cathode material investigated by X-ray absorption spectroscopy. J. Power Sources 174, 1015-1020.

Yoon W S, Haas O, Muhammad S, Kim H, Lee W, Kim D, Fischer D A, Jaye C, Yang X Q, Balasubramanian M, and Nam K W (2014) In situ soft XAS study on nickel-based layered cathode material at elevated temperatures: A novel approach to study thermal stability. Sci. Rep. 4, 6827-6831.

Zhang D, Haran B S, Durairajan A, White R E, Podrazhansky Y, and Popov B N (2000) Studies on capacity fade of lithium-ion batteries. J. Power Sources 91, 122-129.

Zhang J, Chang L, Wang F X, Xie D, Su Q M, and Du G H (2015) Ultrafine $\mathrm{SnO}_{2}$ nanocrystals anchored graphene composites as anode material for lithium-ion batteries. Mater. Res. Bull. 68, 120-125.

Zhang Y, Zhang X G, Zhang H L, Zhao Z G, Li F, Liu C, and Cheng H M (2006) Composite anode material of silicon/graphite/carbon nanotubes for Li-ion batteries. Electrochim. Acta. 51, 4994-5000.

Zou B K, Zhang Y Y, Wang J Y, Liang X, Ma X H, and Chen C H (2015) Hydrothermally enhanced $\mathrm{MnO} /$ reduced graphite oxide composite anode materials for high performance lithium-ion batteries. Electrochim. Acta. 167, 25-31. 